\title{
Method for performing cerebral perfusion-weighted MRI in neonates
}

\author{
Tarek Laswad • Pia Wintermark • Leonor Alamo • \\ Adrien Moessinger $\cdot$ Reto Meuli $\cdot$ François Gudinchet
}

Received: 17 June 2008 /Revised: 2 October 2008 / Accepted: 20 October 2008 / Published online: 23 December 2008

(C) Springer-Verlag 2008

\begin{abstract}
Cerebral perfusion-weighted imaging (PWI) in neonates is known to be technically difficult and there are very few published studies on its use in preterm infants. In this paper, we describe one convenient method to perform PWI in neonates, a method only recently used in newborns. A device was used to manually inject gadolinium contrast material intravenously in an easy, quick and reproducible way. We studied 28 newborn infants, with various gestational ages and weights, including both normal infants and those suffering from different brain pathologies. A signal intensity-time curve was obtained for each infant, allowing us to build perfusion maps. This technique offered a fast and easy method to manually inject a bolus gadolinium contrast material, which is essential in performing PWI in neonates. Cerebral PWI is technically feasible and reproducible in neonates of various gestational age and with various pathologies.
\end{abstract}

Keywords Newborn · Brain · MRI ·

Perfusion-weighted imaging

\section{Introduction}

Cerebral perfusion-weighted imaging (PWI) is known to be a minimally invasive technique and provides data at a

T. Laswad $(\bowtie) \cdot$ L. Alamo $\cdot$ R. Meuli $\cdot$ F. Gudinchet

Radiology Department, Centre Hospitalier Universitaire Vaudois

(CHUV) and University of Lausanne,

1011 Lausanne, Switzerland

e-mail: tarek_laswed@yahoo.com

P. Wintermark $\cdot$ A. Moessinger

Division of Neonatology, Centre Hospitalier Universitaire Vaudois

(CHUV) and University of Lausanne,

1011 Lausanne, Switzerland relatively high resolution allowing direct comparison of the perfusion of different structures within the brain [1]. Many studies on cerebral PWI have been performed in adults using dynamic-susceptibility contrast-enhanced (DSCE) gradientecho MRI. The noninvasive character of PWI, the susceptibility for microvascular haemodynamic alterations, the short acquisition times, the lack of ionizing radiation and the present widespread availability of MRI scanners make PWI ideally suited to children. However, cerebral PWI has only recently been used in newborns and there are very few published studies using PWI in the very immature brain $[1,2]$. The main technical difficulties are the small head size of newborns, fast heart rate, motion artefacts and the small quantity of contrast material to be injected.

Intravenous (IV) infusion of contrast material is known to have risks, such as contrast material extravasation into the soft tissue surrounding the injection site. To obtain cerebral PWI with good quality images an adequate quantity of contrast material injected at a high flow rate is mandatory. The use of power injectors has been associated with higher rates of extravasation than manual injections [3].

We describe here a manual, simple and reproducible technique for IV infusion of gadolinium contrast material for cerebral PWI. We also sought to determine whether PWI is technically feasible in neonates of various gestational ages, weights and pathologies.

\section{Technique}

A prospective study of 28 newborn infants was performed over a period of 2 years (October 2005 to June 2007). Cerebral PWI was added in all infants who underwent a MRI study (conventional MRI, diffusion-weighted imaging and PWI) as part of a detailed clinical neurological evaluation. Parental 
consent was obtained for all injections of gadolinium. Diuresis, serum urea and creatinine levels were available for all patients. The local ethics committee approved the study protocol. The inclusion criterion was an agreed conventional MRI scan as part of a detailed clinical neurological evaluation. The exclusion criteria were impaired renal function, oliguria and infants too unstable for transport to the MRI scanner.

The MRI study was obtained when the patients were stable for transport to the MRI scanner. Newborn infants were wrapped in a pillow containing polystyrene balls. Blankets were wrapped around the infant and the pillow, keeping the infant warm during scanning. Ear muffs were used to reduce the effects of gradient-generated noise. No sedation was used except in a single patient with hypoxicischaemic encephalopathy who manifested a seizure during the MRI scan necessitating IV injection of benzodiazepine.
MRI was performed using a 1.5-T machine (Symphony, Siemens, Erlangen, Germany). Dynamic, T2*, perfusion, gradient-echo EPI images were acquired (TR/TE 1,220/ $54 \mathrm{~ms}$ ) during the injection. Twelve axial sections (slice thickness $5 \mathrm{~mm}$; interslice gap $0.5 \mathrm{~mm}$; field of view $230 \mathrm{~mm}$; matrix size $128 \times 128$ ) were imaged 40 times every $1.26 \mathrm{~s}$. After approximately 15 baseline images had been acquired, an IV bolus of $0.2 \mathrm{ml} / \mathrm{kg}$ of gadolinium contrast material at a dose of $0.1 \mathrm{mmol} / \mathrm{kg}$ (Omniscan; GE Healthcare Bio-Sciences, Little Chalfont, UK), released by the manufacturer for use in newborns at the time of the study, was manually injected. Our device is composed of the following components: one needle (19 gauge; Terumo Medical Corporation, Somerset, NJ), one 1-ml syringe, one 5-ml syringe, a 3-ml tube extension, and normal saline (Fig. 1). The device is assembled by connecting the 5-ml syringe to the $3-\mathrm{ml}$ tube extension with a

Fig. 1 Equipment and assembly. a Materials: from left to right one needle, one 1-ml syringe, one 5-ml syringe, two stopcocks, 3-ml extension tubing, and saline. b Assembly: the 5-ml syringe connected to the 3$\mathrm{ml}$ extension tubing with a stopcock at each end and filled with saline. c Filling with $0.1 \mathrm{mmol} / \mathrm{kg}$ of gadolinium contrast material. d Ready to be connected to the patient. e Ready to perform injection
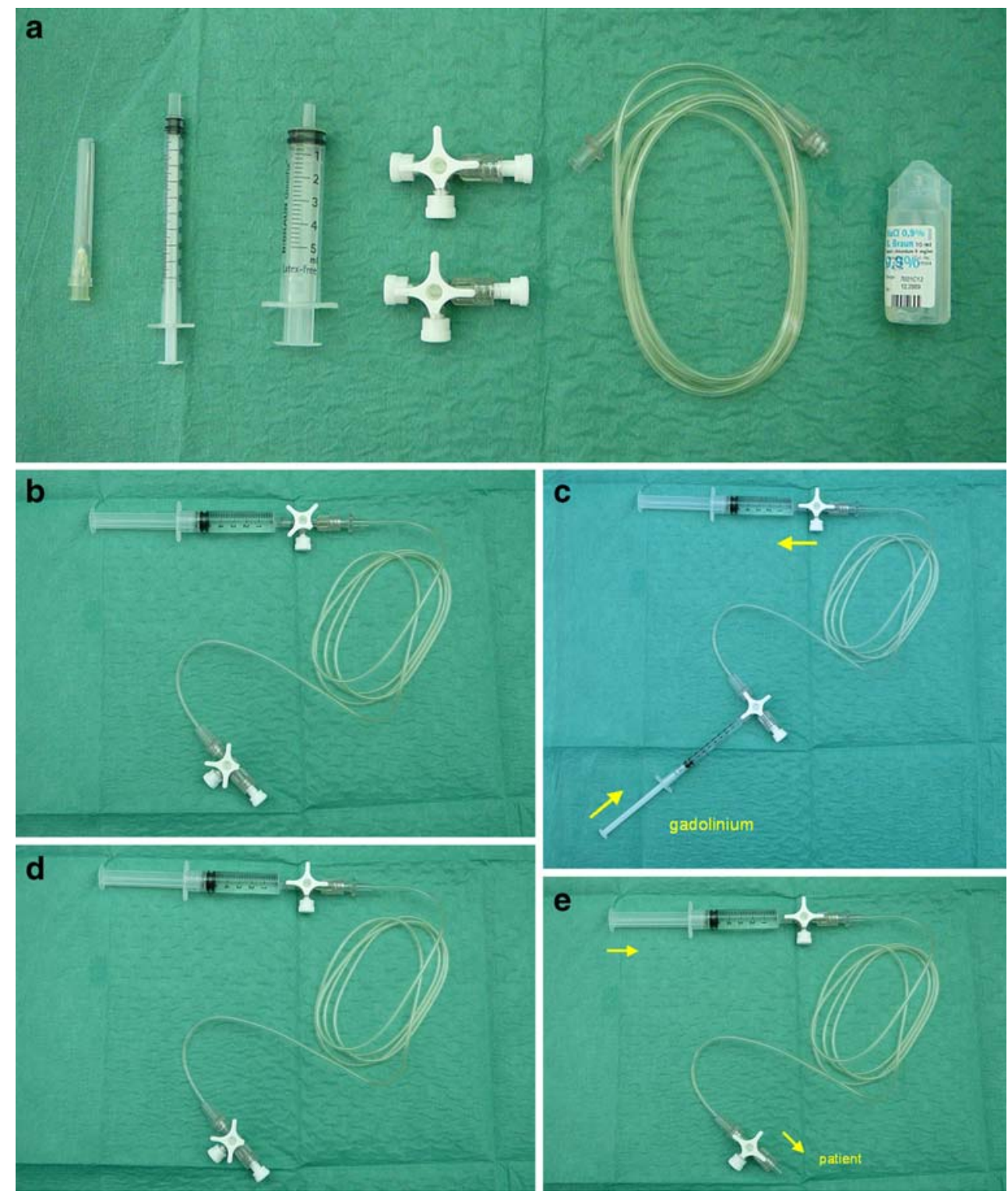


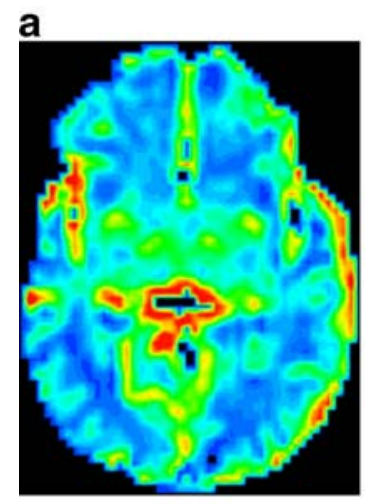

b

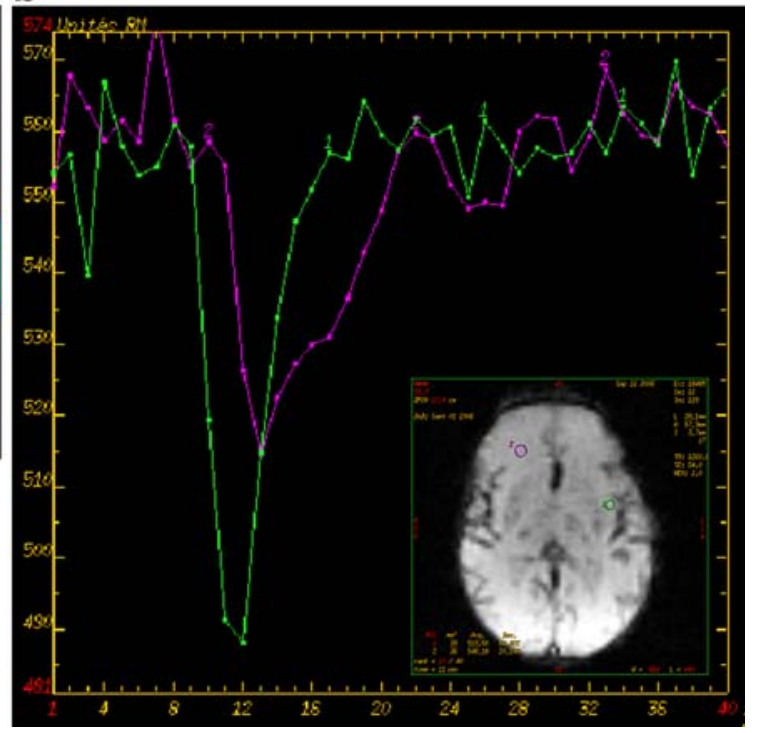

Fig. 2 Normal perfusion in a term newborn infant. a rCBV map reveals symmetrical perfusion of both cerebral hemispheres. Note the physiological distribution of greater blood volume in the basal ganglia. b The concentration-time curve can be used to evaluate

stopcock at each end (Fig. 1). The tube extension is then flushed with normal saline. The tube is slowly filled with $0.1 \mathrm{mmol} / \mathrm{kg}$ of gadolinium contrast material from the other end by the $1-\mathrm{ml}$ syringe and normal saline is withdrawn at the same time into the $5-\mathrm{ml}$ syringe so that the contrast lies in contact with normal saline as demonstrated in Fig. 1. The device is then connected to the venous line of the patient in readiness to perform the bolus injection (Fig. 1). The venous line was a 24- or 26-gauge catheter placed in the dorsum of the hand or in the antecubital fossa.

Complete imaging and data were available for 28 studies in 25 nonsedated newborn infants with a mean gestational age of 39 weeks (range 31-42 weeks) and a mean weight of $2,902 \mathrm{~g}$ (range $815-4,000 \mathrm{~g}$ ). Nine of the 28 studies were normal. Brain pathologies were hypoxic-ischaemic encephalopathy $(n=8)$, stroke $(n=2)$, intracerebral haemorrhage $(n=6)$, congenital infection $(n=2)$, and hemimegalencephaly $(n=1)$. No significant motion artefacts were present.

A signal intensity-time curve was obtained for each patient, permitting us to build perfusion maps. Qualitative perfusion maps were obtained using General Electric Functool Software 2.6.8 (GE Medical System, Milwaukee, WI). The maximum slope of decrease and negative enhancement integral were used as qualitative relative cerebral blood flow $(\mathrm{rCBF})$ and relative cerebral blood volume (rCBV) maps.

The arterial input function (AIF) for each patient was obtained by manual selection of pixels in the neighbourhood of the middle cerebral artery or internal carotid artery. The rCBF was obtained as the maximum value of the residue function obtained by SVD (singular value decomposition). The rCBV was obtained by integrating the concentration-time relative blood volume. Note the greatest blood volume (purple curve) found in the cortical mantle compared to the lower blood volume (green curve), as expected, in the white matter region

curves between selected time points used (typically corresponding to the arrival of contrast agent in the region of interest and the end of the first pass).

The perfusion maps obtained displayed perfusion variations in the different normal brain regions, such as the

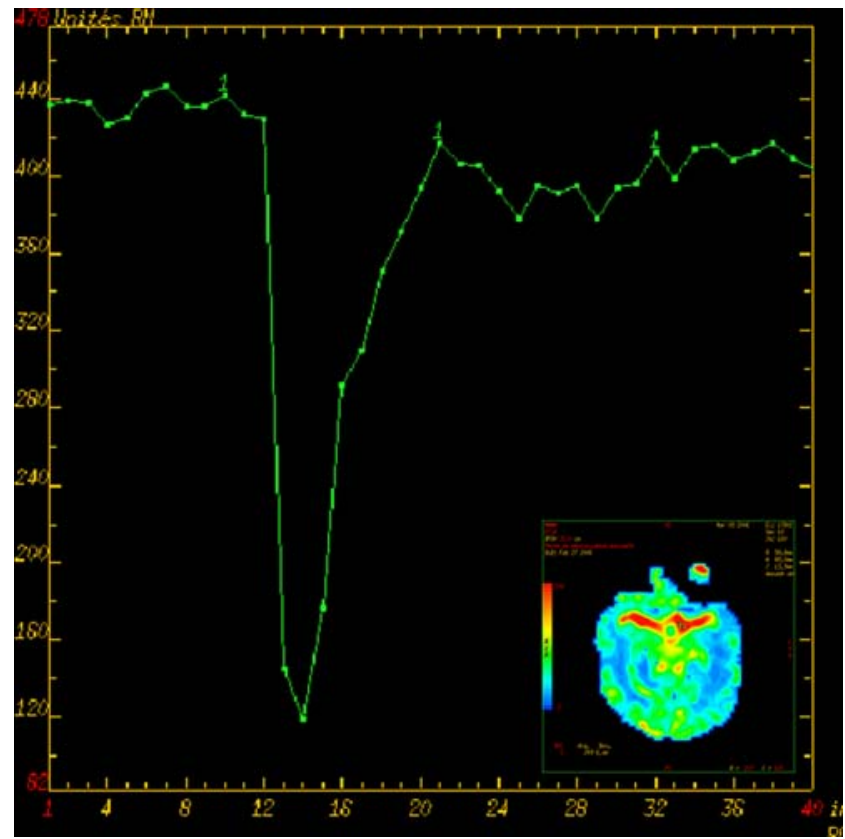

Fig. 3 Concentration-time curve in a voxel within the left internal carotid artery used as an arterial input function. The curve shows the sharp decline in signal intensity on contrast medium arrival and the sharp increase with wash-out of the contrast medium. The signal intensity remains slightly lower than the initial baseline signal intensity due to residual circulating contrast agent 
physiologically increased perfusion in the basal ganglia and cortex compared to white matter (Fig. 2). They also displayed the perfusion abnormalities associated with several brain pathologies affecting newborns.

\section{Discussion}

Abnormalities of cerebral perfusion are believed to be of prime importance in a number of basic pathologies associated with the neonatal and infant brain. Perfusionweighted MRI is technically feasible and reproducible in neonates of various gestational ages and pathologies. It involves the administration of gadolinium, but is otherwise noninvasive and could, therefore, be easily included as part of a clinically indicated MRI scan.

To perform cerebral PWI the fast and safe injection of an IV bolus of gadolinium contrast material is essential. Many methods of IV injection of contrast material have been described. Automated power injectors were developed for injection of contrast material through peripheral venous catheters at specific flow rates. However, their most important complication is the risk of extravasation of contrast material. This risk is more important in children than adults [4]. The use of power injectors in the imaging of children is, therefore, avoided in some institutions. Powel et al. [5] described a new device (extravasation detection accessory) to limit extravasation during contrast-enhanced CT studies performed with power injectors. Other traditional manual or drip injection methods may not provide optimum flow rates, and peak levels of organ enhancement may not always be achieved [6].

Our device permits the manual injection of gadolinium in an easy, quick and reproducible manner. It does not require additional larger access for bolus delivery than those already in place in a newborn coming for an MRI study. In all our cases filling of the tube with gadolinium was performed at the beginning of the examination and hooked up to the patient at the beginning of the MRI study. Thus we did not have to move the patient during the MRI examination. The 3-ml extension tubing was filled very slowly with gadolinium in order to avoid mixing of normal saline and gadolinium.

Some degree of contrast dilution is inevitable. Nevertheless, thanks to the small volume of the extension tubing ( $3 \mathrm{ml}$ ) and the stopcock at each end, the dilution of gadolinium is very limited. For example, a $3-\mathrm{kg}$ child needing $0.6 \mathrm{ml}$ of contrast medium has that contrast medium dose diluted only by a factor of 5 . Therefore, the limited dilution allows a short enough bolus injection for adequate perfusion data acquisition. It allows fast injection of contrast material flushed by $5 \mathrm{ml}$ of normal saline, permitting a sharp bolus profile that enables the generation of accurate rCBF, rCBV and transit time PWI maps (Figs. 2 and 3). In a recent study, Wintermark et al. [7] used the same device to perform cerebral PWI for the evaluation of the temporal evolution of neonatal hypoxic-ischaemic encephalopathy in two newborn infants and three control infants.

In our series, the quality of bolus IV injection of gadolinium contrast material allowed correct diagnoses in all patients with pathology and the realization of qualitative perfusion maps in all patients. Comparing the three normal patients who were examined using MRI scans including PWI, values of CBF in the different regions of interest were similar. Furthermore, in four patients with hypoxic-ischaemic encephalopathy MRI scans including PWI were repeated early and late after birth. Values of perfusion in the cerebral regions where there were no hypoxic-ischaemic lesions were similar between the early and late scans.

The obtained perfusion maps in all of our patients displayed sufficient resolution to detect perfusion differences in the different normal brain regions and also to detect perfusion abnormalities in newborns with pathologies involving brain vessels.

Recent warnings suggest that gadolinium contrast agent should be used cautiously in patients with renal function disturbance. It is therefore imperative that paediatric practitioners are prudent and immediately tighten their local policies on the use of IV gadolinium-based contrast agents [8]. The manufacturers have extended these precautions to neonates in view of their immature renal function. It is important to state that all our patients were studied before these warnings were issued. Nevertheless, no early or late adverse effects have been documented so far.

In conclusion, we demonstrated that this method for the manual injection of contrast medium is easy, quick and safe, and allows reproducible PWI maps to be obtained in the neonatal age group. Further studies with increased numbers of patients will bring more information about normal values of brain perfusion in newborn infants.

\section{References}

1. Tanner SF, Cornette L, Ramenghi LA et al (2003) Cerebral perfusion in infants and neonates: preliminary results obtained using dynamic susceptibility contrast enhanced magnetic resonance imaging. Arch Dis Child Fetal Neonatal Ed 88:F525F530

2. Rutherford M, Ward P, Allsop J et al (2005) Magnetic resonance imaging in neonatal encephalopathy. Early Hum Dev 81:13-25

3. Huisman TA, Sorensen AG (2004) Perfusion-weighted magnetic resonance imaging of the brain: techniques and application in children. Eur Radiol 14:59-72

4. Amaral JG, Traubici J, BenDavid G et al (2006) Safety of power injector use in children as measured by incidence of extravasation. AJR 187:580-583 
5. Powell CC, Li JM, Rodino L et al (2000) A new device to limit extravasation during contrast-enhanced CT. AJR 174:315318

6. Sistrom CL, Gay SB, Peffley L (1991) Extravasation of iopamidol and iohexol during contrast-enhanced CT: report of 28 cases. Radiology 180:707-710
7. Wintermark P, Moessinger AC, Gudinchet F et al (2008) Temporal evolution of MR perfusion in neonatal hypoxic-ischemic encephalopathy. J Magn Reson Imaging 27:1229-1234

8. Mendichovszky IA, Marks SD, Simcock CM et al (2008) Gadolinium and nephrogenic systemic fibrosis: time to tighten practice. Pediatr Radiol 38:489-496; quiz 602-603 